\title{
ELEVADAS MUDANÇAS DE CURTO PRAZO E HETEROGENEIDADE INTRALAGUNAR NA EMISSÃO DE AMÔNIA DE UMA LAGOA COSTEIRA URBANA TROPICAL (LAGOA RODRIGO DE FREITAS - RIO DE JANEIRO) À ATMOSFERA
}

\author{
Maria Carolina Barroso dos Santos ${ }^{1}$, Humberto Marotta ${ }^{2}$ \& Alex Enrich-Prast $^{1,3}$ \\ ${ }^{1}$ Universidade Federal do Rio de Janeiro (UFRJ), Instituto de Biologia, Laboratório de Biogeoquímica, Departamento de Ecologia, Ilha do Fundão, \\ Caixa Postal: 68020. Rio de Janeiro, RJ, Brasil. CEP: 2151-290. \\ ${ }^{2}$ Universidade Federal Fluminense (UFF), Instituto de Geociências, Laboratório de Processos Sedimentares e Ambientais (LAPSA/UFF), Departmento \\ de Geografia, Niterói, RJ, Brasil. CEP: 24210-340. \\ ${ }^{3}$ Endereço atual: Department of Thematic Studies - Water and Environmental Studies, Linkoping University, Linkoping, Sweden. \\ Emails: mcbs_rj@ibest.com.br, humbertomarotta@gmail.com, aenrichprast@gmail.com.
}

\section{RESUMO}

A eutrofização e as emissões de gases do efeito estufa em ecossistemas aquáticos continentais são problemas estreitamente relacionados com a produção e emissão de nitrogênio $(\mathrm{N})$ em muitas formas, especialmente as formas inorgânicas que tem sido fortemente aumentada pelo uso das bacias hidrográficas e do solo pelo homem. Quantidades de $\mathrm{N}$ amoniacal, como amônia inorgânica $\left(\mathrm{NH}_{3}\right)$ e amônio $\left(\mathrm{NH}_{4}^{+}\right)$, são liberadas na decomposição orgânica e pode ter implicações ecológicas em áreas altamente produtivas e lagoas costeiras que recebem aportes autóctones terrestres e insumos orgânicos. O objetivo desse estudo foi avaliar as mudanças em curto prazo e a variabilidade intralagunar para as emissões de amônia a partir de uma lagoa costeira tropical urbana (Rodrigo de Freitas) para a atmosfera. Os fluxos de amônia variaram intensamente de 1.2 a $3500 \mu \mathrm{gN} \mathrm{m}^{-2} \mathrm{~h}^{-1}$ entre as estações amostradas e ao longo dos dias. A estação mais eutrófica (próxima ao Jockey Clube do Rio de Janeiro), é também a que possui os maiores valores de emissão de amônia, variando em mediana ( \pm interquartil 25-75) 270.1 $\pm 150-976 \mu \mathrm{g} \mathrm{N} \mathrm{m}^{-2} \mathrm{~h}^{-1}$, ou seja, valores 50 vezes maiores do que os observados nas outras estações. Aumentos drásticos nas emissões de amônia foram relatados com eventos de precipitação, variando 700 vezes de 1.2-5 $\mu \mathrm{g} \mathrm{N} \mathrm{m}^{-2} \mathrm{~h}^{-1}$ antes da chuva para $3500 \mu \mathrm{g} \mathrm{N} \mathrm{m}-{ }^{2} \mathrm{~h}^{-1}$ nos dias chuvosos. As emissões de amônia em águas superficiais de lagoas costeiras tropicais urbanas podem ser altamente dinâmicas, mostrando drásticas mudanças de curto prazo e heterogeneidade intralagunar alta, com implicações importantes para as emissões de $\mathrm{N}$ a partir de extensas áreas inundadas para a atmosfera.

Palavras-chave: nitrogênio; mudanças de curto prazo; heterogeneidade intralagunar; emissão de amônia; lagoas costeiras tropicais.

\begin{abstract}
DRASTIC SHORT-TERM CHANGES AND INTRA-LAKE HETEROGENEITY IN AMMONIUM EMISSIONS FROM A TROPICAL URBAN COASTAL LAGOON (RODRIGO DE FREITAS, RIO DE JANEIRO) TO THE ATMOSPHERE. Eutrophication and emissions of greenhouse gases in continental aquatic ecosystems are a problem closely related to production and emission of nitrogen $(\mathrm{N})$ in many forms, especially those inorganic that have been strongly increased by the human land use in the watershed. Ammoniacal $\mathrm{N}$ amounts, as inorganic ammonia $\left(\mathrm{NH}_{3}\right)$ and ammonium $\left(\mathrm{NH}_{4}^{+}\right)$, are released by the organic decomposition and may have important ecological implications in highly organic and productive coastal lagoons receiving terrestrial or autochthonous organic inputs. The aim of this study was to assess short-term ckanges and the intralake herterogeneity for ammonia emissions from a tropical coastal urban lagoon (Rodrigo de Freitas) to the atmophere. ammonia fluxes varied intensely from 1.2 to $3500 \mu \mathrm{g} \mathrm{N} \mathrm{m}^{-2} \mathrm{~h}^{-1}$ among sampling stations and over the daytime The most eutrophic station (next to the racetrack of Rio de Janeiro), the also showed higher values
\end{abstract}


of ammonia evasion, in average ( \pm standard error), $1052 \pm 188.6 \mu \mathrm{g} \mathrm{N} \mathrm{m}^{-2} \mathrm{~h}^{-1}$, or about 50 times higher than those observed in other stations. Drastic increases in ammonia emissions were reported with rainfall events, varying 700 times from $1.2-5 \mu \mathrm{g} \mathrm{N} \mathrm{m}-{ }^{2} \mathrm{~h}^{-1}$ before to $3500 \mu \mathrm{g} \mathrm{N} \mathrm{m}-{ }^{2} \mathrm{~h}^{-1}$ in rainy days. Ammonia in surface waters of urban tropical coastal lagoons may be highly dynamic, showing drastic short-term changes and high intralake heterogeneity with important implications for $\mathrm{N}$ emissions from extensive flooded areas to the atmosphere.

Keywords: nitrogen; short-term changes; intralake heterogeneity; ammonia emissions; tropical coastal lagoons.

\section{RESUMEN}

CAMBIOS DRÁSTICOS A CORTO PLAZO Y HETEROGENEIDAD INTRALAGUNAR EN LA EMISIÓN DE AMONIO DE UNA LAGUNA COSTERA URBANA TROPICAL (LAGUNA RODRIGO DE FREITAS - RIO DE JANEIRO) A LA ATMÓSFERA - La eutrofización y las emisiones de gases de efecto invernadero en ecosistemas acuáticos continentales son problemas estrechamente relacionados con la producción y emisión de nitrógeno $(\mathrm{N})$ en muchas formas, especialmente en formas inorgánicas, que han aumentado fuertemente debido al uso de cuencas hidrográficas y del suelo por el hombre. Cantidades de $\mathrm{N}$ amoniacal, como amoníaco $\left(\mathrm{NH}_{3}\right)$ y amonio $\left(\mathrm{NH}_{4}^{+}\right)$, son liberadas durante la descomposición orgánica lo cual puede tener implicaciones ecológicas en áreas altamente productivas y lagunas costeras que reciben aportes autóctonos terrestres e insumos orgánicos. El objetivo del presente estudio fue evaluar los cambios a corto plazo y la variabilidad intralagunar en las emisiones de amonio de una laguna costera tropical urbana (Rodrigo de Freitas) a la atmósfera. Los flujos de amonio variaron fuertemente, de $1.2 \mathrm{a} 3500 \mu \mathrm{gN} \mathrm{m}^{-2} \mathrm{~h}^{-1} \mathrm{entre}$ las estaciones muestreadas y a lo largo de los días. La estación más eutrófica (próxima al Jockey Club de Río de Janeiro), es también la que presentó los mayores valores de emisión de amonio, variando en mediana ( \pm intercuartil 25-75) $270.1 \pm 150-976 \mu \mathrm{g} \mathrm{N} \mathrm{m}^{-2} \mathrm{~h}^{-1}$, o sea, valores 50 veces mayores que los observados en las otras estaciones. Aumentos drásticos en las emisiones de amonio estuvieron relacionados a eventos de precipitación, siendo 700 veces mayor, de $1.2-5 \mu \mathrm{g} \mathrm{N} \mathrm{m}^{-2} \mathrm{~h}^{-1}$ antes de la lluvia a $3500 \mu \mathrm{g} \mathrm{N} \mathrm{m}-{ }^{2} \mathrm{~h}^{-1}$ en los días lluviosos. Las emisiones de amonio de aguas superficiales de lagunas costeras tropicales urbanas pueden ser altamente dinámicas, mostrando cambios drásticos a corto plazo y heterogeneidad intralagunar alta, con implicaciones importantes para las emisiones de $\mathrm{N}$ de extensas áreas inundadas a la atmósfera.

Palabras clave: nitrógeno; cambios a corto plazo; heterogeneidad intralagunar; emisión de amonio; lagunas costeras tropicales.

\section{INTRODUÇÃO}

O nitrogênio $(\mathrm{N})$ é um dos principais nutrientes limitantes da atividade biológica, cujo aporte antropogênico tem se intensificado sensivelmente nas últimas décadas (Vitousek et al. 1997). Este nutriente limita a entrada de energia dos ecossistemas e constitui um componente essencial da biomassa dos seres vivos (Esteves 1998). No entanto, seu excesso também pode causar a degradação da qualidade da água, eutrofização, diminuição do uso humano e a redução da diversidade biológica (Johnson et al. 2008). O aporte de N inorgânico pode favorecer tanto a decomposição orgânica que libera gases de carbono causadores do aquecimento global por efeito estufa (Morris \& Bradley 1999) quanto à produção primária que apreende parte desses gases. Além disso, a mineralização da matéria orgânica também pode redisponibilizar os próprios nutrientes inorgânicos ao meio, que incluem o $\mathrm{N}$ amoniacal (Dentener 1994) sob as formas de amônia $\left(\mathrm{NH}_{3}\right)$ e íon amônio $\left(\mathrm{NH}_{4}^{+}\right)$(Fenchel 2008). O N amoniacal é formado pelo processo de amonificação, no qual o nitrogênio orgânico no ambiente aquático é mineralizado à amônia por bactérias e fungos (Leal et al. 2005).

Nos ecossistemas aquáticos, a disponibilidade relativa de amônia ou íon amônio no estoque total de $\mathrm{N}$-amoniacal depende do $\mathrm{pH}$ das águas, uma vez que condições mais ácidas tendem a apresentar maior proporção de $\mathrm{NH}_{4}^{+}$dissolvido, enquanto em condições básicas há mais $\mathrm{NH}_{3}$. $\mathrm{O}$ fluxo de determinado gás na interface água-atmosfera é resultante primariamente 
do desequilíbrio das concentrações gasosas entre ambos os meios. Essa diferença de concentração de determinado gás é consequência de processos biológicos e químicos relacionados à produção e ao consumo do gás ou, ainda, de variações nos parâmetros físico-químicos que influenciam sua solubilidade e taxa de transferência à atmosfera, especialmente temperatura, salinidade, pressão e velocidade do vento (Quinn et al. 1996). Em razão de sua propriedade altamente volátil e de reatividade química, elevadas concentrações de $\mathrm{NH}_{3}$ podem apresentar diversas implicações ecológicas. O excesso de amônia pode acidificar as águas, contribuindo para o fenômeno da chuva ácida na atmosfera, ou ainda alcançar elevados níveis tóxicos para fauna e flora (Felix \& Cardoso 2004). Apesar de ser encontrado em quantidades menores em relação aos demais gases atmosféricos, $\mathrm{o} \mathrm{NH}_{3}$ é a principal base gasosa presente no ar, possuindo um tempo de residência na atmosfera de 1 a 2 semanas e elevada reatividade com ácidos presentes, tais como ácido nítrico e ácido sulfúrico (Felix \& Cardoso 2004).

A supersaturação de $\mathrm{NH}_{3}$ e sua subsequente emissão dos lagos à atmosfera pode ser potencialmente relevante em escala global (Johnson et al. 2008). Os lagos tropicais apresentam elevadas temperaturas o ano inteiro, de forma que os processos metabólicos são intensos e variáveis (Marotta et al. 2009). O elevado dinamismo tropical dos processos envolvidos na decomposição orgânica pode ser ainda mais acentuado nas lagoas costeiras (Marotta et al. 2010a, Marotta et al. 2010b), ecossistemas frequentemente rasos que são separados do oceano por cordões litorâneos orientados paralelamente a linha de costa (Kjerfve \& Magill 1989). As lagoas costeiras estão sujeitas a intensas mudanças de curto prazo nos fatores meteorológicos e no ciclo de maré, resultando em variações na precipitação ou na entrada de água marinha que representam importantes condições e recursos à biota (Marotta et al. 2010a). Além disso, essas lagoas podem apresentar alto grau de heterogeneidade intra-lagunar (Tundisi 1997), os quais aumentam potencialmente a variação dos processos biológicos (Kolasa \& Rollo 1991). Apesar de pouco estudadas, flutuações diárias e semanais dos nutrientes orgânicos e inorgânicos (Yannarell et al. 2003) e de gases envolvidos na decomposição orgânica (Staehr and Sand-Jensen 2007) podem ser especialmente relevantes nas águas interiores. Uma das alterações humanas mais frequentes na bacia de drenagem é o aporte de efluentes domésticos que resulta no enriquecimento de nutrientes (eutrofização) das águas lacustres (Schindler et al. 1997, Schindler 2006, Marotta et al. 2008). Essa dinâmica também é comum na costa brasileira (Esteves 1998). Os efluentes domésticos são ricos tanto em nutrientes inorgânicos quanto em compostos orgânicos, favorecendo o processo de mineralização da matéria orgânica (Marotta et al. 2010a). Além disso, as altas temperaturas, a pluviosidade, as variações das condições físicas e da disponibilidade de nutrientes nos lagos tropicais eutrofizados intensificam a mineralização da matéria orgânica a nutrientes e a gases com a propriedade de intensificar o aquecimento global (Tranvik et al. 2009), os quais incluem as formas de $\mathrm{N}$ amoniacal (Asman et al. 1998).

Apesar desta potencial importância ecológica associada à alta capacidade de dispersão da amônia na atmosfera (Sutton et al. 1998), ainda são escassos os estudos sobre as emissões deste composto pelos ecossistemas aquáticos (Guimaraes \& De Mello 2006), especialmente ao longo de escalas de tempo de curta duração. O objetivo desse estudo foi avaliar as variações de curto prazo entre cinco dias consecutivos e na escala espacial intra-lagunar nas emissões de amônia de uma lagoa costeira tropical urbana (Lagoa Rodrigo de Freitas) à atmosfera.

\section{MATERIAL E MÉTODOS}

\section{ÁREA DE ESTUDO}

A Lagoa Rodrigo de Freitas é um ecossistema costeiro tropical localizado na zona sul da cidade do Rio de Janeiro (latitude: $22^{\circ} 57^{\prime} 02^{\prime \prime}-22^{\circ} 58^{\prime} 09^{\prime} \mathrm{S}$; longitude: $043^{\circ} 11^{\prime} 09^{\prime \prime}-043^{\circ} 13^{\prime} 03^{\prime \prime} \mathrm{W}$ (Figura 1). Possui $3 \mathrm{~km}$ de extensão em sua maior largura $7,2 \mathrm{~km}$ de perímetro e área total de $2,5 \mathrm{~km}^{2}$ (Oliveira 1976). Apresenta um volume de água de aproximadamente $6.200 .000 \mathrm{~m}^{3}$ (Bouwman et al. 1997) e profundidade média em torno de 2,8m (Machado 2008). É uma lagoa urbana, rasa e submetida aos aportes esporádicos de esgoto e de águas salobras (Pereira 2009). A bacia hidrográfica da Lagoa Rodrigo de Freitas possui uma área de cerca de $20 \mathrm{~km}^{2}$, sendo seus principais contribuintes os rios dos Macacos, Cabeça e Rainha. 
A Lagoa Rodrigo de Freitas pode ser classificada como lagoa sufocada, pois apresenta uma única via de comunicação com o mar, o canal do Jardim de Alah que possui cerca de $800 \mathrm{~m}$ de comprimento e de 10 a 18 metros de largura (Pereira 2009). A ineficiente troca de águas com o mar, devido ao intenso assoreamento do canal, tem causado o aumento do tempo de residência das águas, de forma a resultar no acúmulo de materiais em suspensão e matéria orgânica na lagoa (Araujo 2008).

Já o clima da área de estudo é tropical, quente e úmido, caracterizado por uma temperatura média anual de $22^{\circ} \mathrm{C}$ e umidade acima dos $78 \%$ (Machado 2008). A média anual de pluviosidade também é elevada, em torno de $1300 \mathrm{~mm}$, apresentando o período mais chuvoso de Janeiro a Março e uma curta estação seca tipicamente entre Junho e Setembro (dados do Instituto Nacional de Metereologia - INMET- entre 2006 e 2011).

\section{ESTAÇÕES DE COLETA}

Foram amostradas simultaneamente cinco estações que fazem parte do monitoramento mensal do Instituto Estadual de Meio Ambiente na lagoa Rodrigo de Freitas, cujas nomenclaturas designadas por este órgão também foram mantidas (LRF00,
LRF01, LRF04, LRF05 e LRFJA; Figura 1). LRF00 (22 $58^{\prime} 15,76^{\prime \prime}$ ' $\left.; 43^{\circ} 12^{\prime} 40,53^{\prime \prime} \mathrm{W}\right)$ representa a porção central da lagoa, onde há menos barreiras físicas para o deslocamento do vento. LRF01 (22 ${ }^{\circ} 57^{\prime} 56,33^{\prime \prime}$ 'S; $\left.43^{\circ} 12^{\prime} 42,07^{\prime \prime} \mathrm{W}\right)$ se encontra mais distante do mar, apresentando profundidades de $2-3 \mathrm{~m}$ e entrada de águas pluviais, as quais recebem esgotos in natura procedentes de ligações irregulares e extravasores das elevatórias de esgoto (FEEMA 2006). Já a porção próxima às comportas dos rios Cabeça e dos Macacos,

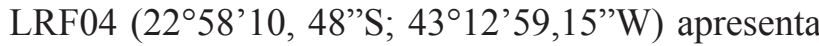
reduzida profundidade devido ao acentuado aporte de sedimentos carreados por esses canais fluviais advindos de áreas densamente povoadas (FEEMA 2006). Essa estação apresenta comumente mistura de águas pluviais e fluviais com efluentes orgânicos do Jóquei Clube e esgotos domésticos (Machado 2008), de forma que sua salinidade é controlada pela abertura das comportas e pela subsequente vazão dos rios que nela aportam(Loureiro 2006). Nooutro extremo oposto ao mar, LRF05 (22 58'33,17’S; 4312'13,39”W) é a estação mais próxima da margem, cuja profundidade é de até $3 \mathrm{~m}$ e também há lançamentos de descargas pluviais contaminadas por esgotos. Finalmente, a

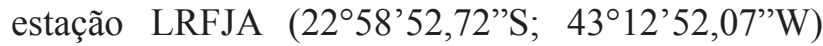
está localizada mais próxima ao mar e ao Canal do Jardim de Alah, que atravessa o cordão litorâneo entre

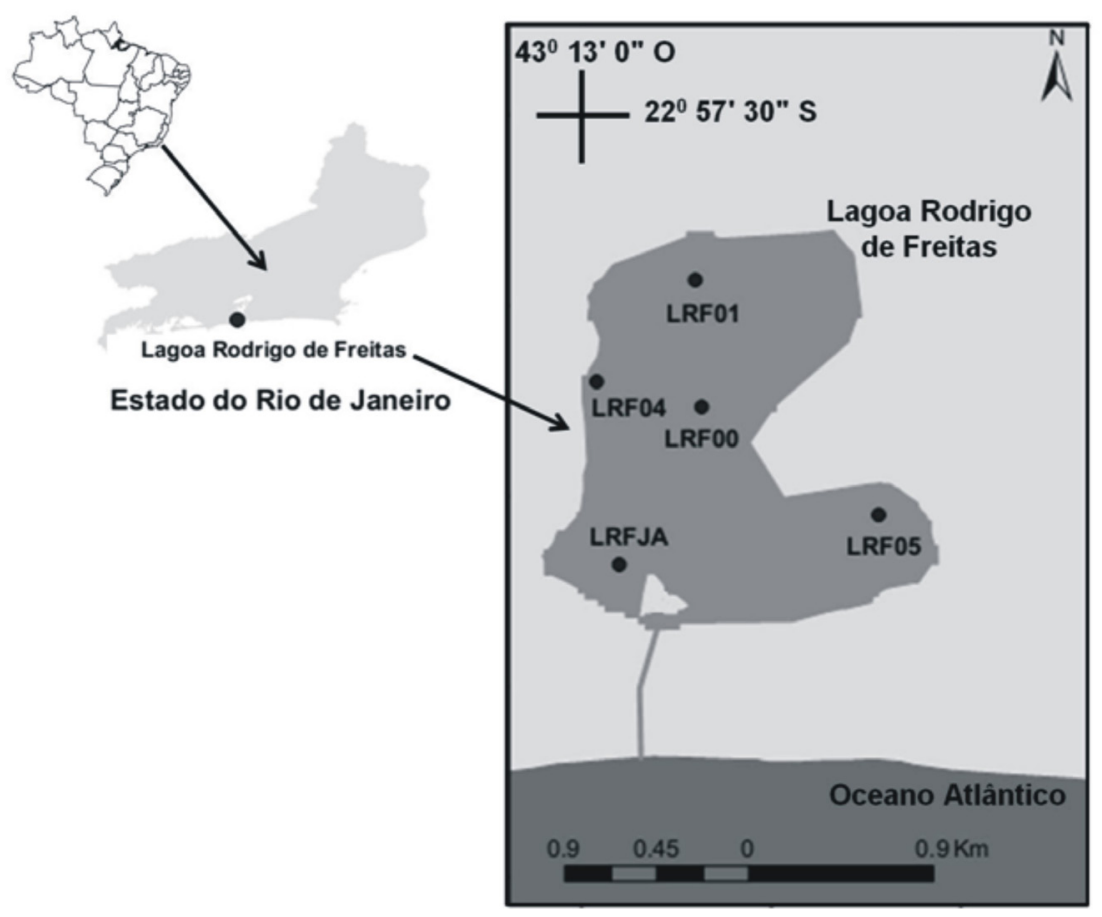

Figura 1. Localização geográfica das estações de coleta na Lagoa Rodrigo de Freitas. Figure 1. Geographic location of sampling stations in Rodrigo de Freitas Lagoon. 
as praias de Ipanema e Leblon. Nessa estação, a troca de massas de água entre a Lagoa e o mar é restrita, embora resulte comumente nos mais acentuados níveis de salinidade e oxigênio da lagoa (Pereira 2009). O estudo foi realizado após 28 dias sem precipitação significativa (pluviosidades entre 0-1 mm; dados do INMET/Alerta Rio - Prefeitura do Rio).

\section{DELINEAMENTO DO ESTUDO}

Amostras de água sub-superficial foram coletadas para análises de clorofila- $\underline{a}$, nitrogênio total (NT), nitrogênio dissolvido (ND), fósforo total (PT) e fósforo dissolvido (PD) nas cinco estações de coleta (Figura 2) ao longo de variação diária (06:00h, 12:00 h e 18:00h) entre 01 a 05 de agosto de 2011. Além disso, $\mathrm{pH}$, salinidade, temperatura, velocidade do vento, umidade relativa do ar, transparência e profundidade total $\left(\mathrm{PROF}_{\text {total }}\right)$ foram mensurados in situ em todos os horários e estações de coleta. No período estudado, a comporta do canal que liga a lagoa ao mar se manteve aberta, de forma que as amplitudes de maré variaram de 0 a $1,5 \mathrm{~m}$ em relação ao nível médio do mar e o horário de maior amplitude média de maré foi entre 03:00 e 04:00h (dados da Diretoria de Hidrografia e Navegação - DHN).
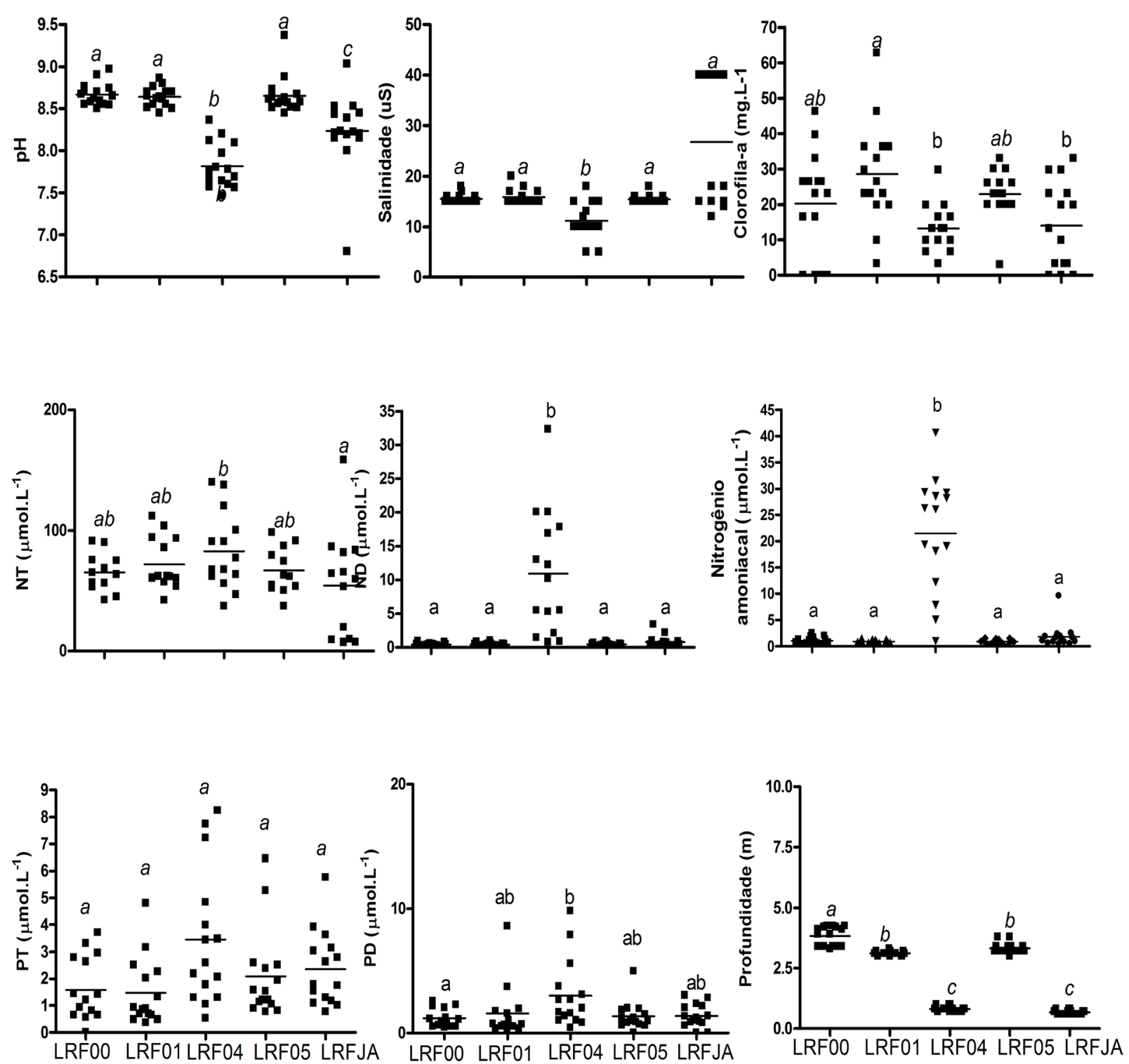

Figura 2. Variáveis físico-químicas nas cinco estações de coleta (LRF00,LRF01,LRF04,LRF05 e LRFJA) de 01 a 05 de agosto de 2011 . O símbolo (匹) representa cada amostra e letras iguais indicam ausência de diferença significativa entre as estações de coleta ( $\mathrm{p}>0,05$, teste de Tukey-Kramer). Figure 2. Physical-chermical variables in the 5 sampling stations (LRF00,LRF01,LRF04,LRF05 and LRFJA) from $1^{\text {st }}$ to $5^{\text {th }}$ August, 2011.The symbol (a) represents each sample and the same letters indicate non-significant differences among sampling stations ( $p>0,05$, Tukey-Kramer test). 


\section{MÉTODOS ANALÍTICOS}

No campo, as concentrações de oxigênio dissolvido (OD) foram fixadas para posterior quantificação pelo método de Winkler em titulador eletrônico (Methron, Tritino plus). $\mathrm{O} \mathrm{pH}$ foi mensurado in situ usando um pHmetro Methron portátil 826, enquanto a temperatura e a salinidade por um termosalinômetro YSI-30 pré-calibrados. Águas subsuperficiais foram coletadas com auxílio de uma seringa de $60 \mathrm{ml}$ e imediatamente condicionadas em frascos de polipropileno para congelamento e posterior análises de nutrientes totais (nitrogênio e fósforo). Outra parte das amostras de água foram previamente filtradas (filtro $0,7 \mathrm{~mm}$, Whatmann $\mathrm{GF} / \mathrm{F}$ ) para análises de nitrogênio e fósforo dissolvidos. As concentrações de fósforo foram determinadas pelo método do azul de molibdênio com pré-digestão ácida e as de nitrogênio pela metodologia de Kjeldahl (Apha 1992), usando um cromatógrafo de íons Methron 850 Professional IC. Já as amostras para $\mathrm{N}$ amoniacal foram previamente filtradas e congeladas in situ, sendo posteriormente analisadas em laboratório pela reação de Berthelot e leitura dos comprimentos de onda de $630 \mathrm{~nm}$ no espectrofotômetro PerkinElmer Lambda XLS.

Análises de clorofila- $\underline{a}$ foram realizadas no particulado retido nos filtros $(0,7 \mathrm{~mm}$ Whatmann GF/F) após filtração de $50 \mathrm{ml}$ de amostra e acondicionamento em criotubos submersos em nitrogênio liquido. No laboratório, as amostras foram extraídas com acetona e lidas nos comprimentos de onda de $433 \mathrm{~nm}$ para medidas da excitação e $673 \mathrm{~nm}$ da emissão (Nusch \& Palme 1975) em um espectrofotômetro PerkinElmer Lambda XLS.

Em relação aos dados meteorológicos, a pluviosidade disponibilizada pelo INMET (Alerta Rio - Prefeitura do Rio) foram agregados pela soma acumulada nas 24 horas que precediam cada evento de coleta. Já a velocidade do vento foi mensurada instantaneamente em cada horário de coleta utilizando uma estação metereológica portátil - Weather Station (receiver) modelo WH1080.

\section{CÁLCULO DO FLUXO DE AMÔNIA}

O fluxo de amônia foi calculado a partir das concentrações de $\mathrm{N}$ amoniacal em um modelo físicoquímico no qual a troca do gás é realizada entre duas camadas adjacentes na interface ar-água. Nesse modelo, assume-se o pressuposto de que ambos os fluídos são bem misturados, tornando a resistência de ambas as fases restrita a uma pequena camada onde a troca é realizada por difusão molecular ao transporte, segundo a fórmula abaixo (Guimarães \& de Melo 2006).

Onde:

$$
\mathbf{F}=\mathbf{k}_{\mathrm{a}}\left(\mathbf{c}_{\mathrm{eq}}-\mathbf{c}_{\mathrm{ar}}\right)
$$

$\mathrm{F}=$ fluxo de amônia $\left(\mu \mathrm{g} \mathrm{m}^{-2} \mathrm{~s}^{-1}\right)$

$\mathrm{k}_{\mathrm{a}}=$ velocidade de transferência em fase gasosa $(\mathrm{m}$

$\left.\mathrm{s}^{-1}\right)$

$\mathrm{c}_{\mathrm{eq}}=$ concentração teórica de amônia no ar em equilíbrio com a concentração medida de nitrogênio amoniacal $\left(\mathrm{NH}_{\mathrm{X}}=\mathrm{NH}_{3}+\mathrm{NH}_{4}^{+}\right)$

$\mathrm{c}_{\mathrm{ar}}=$ concentração de amônia medida no ar, assumida em $5 \mu \mathrm{gm}^{-2} \mathrm{~h}^{-1}$, valor comumente observado nas áreas urbanas (Duce et. al. 1991).

Já o Ceq foi calculado seguindo a fórmula abaixo (Johnson et. al. 2008):

$$
\mathbf{C e q}=\mathbf{M}\left[\mathrm{NH}_{\mathbf{x}}\right] / \mathbf{R T H}_{\mathrm{NH} 3}\left\{\left[1 / \gamma_{\mathrm{NH} 3}\right]+\left[10-\mathbf{p H} /\left(\gamma_{\mathrm{NH} 4} \mathbf{k}_{\mathrm{NH} 4}\right)\right]\right\}
$$

Onde:

$\mathrm{M}=$ massa molecular de $\mathrm{NH}_{3} /\left[\mathrm{NH}_{\mathrm{X}}\right]=$ concentração de nitrogênio amoniacal

$\mathrm{R}=$ constante dos gases perfeitos

$\mathrm{T}=$ temperatura da água

$\mathrm{H}=$ constante da Lei de Henry

$\gamma_{\mathrm{NH} 3}$ e $\gamma_{\mathrm{NH} 4}=$ coeficiente de atividade da amônia e do íon amônio respectivamente na água (o coeficiente é medido a partir da força iônica que é calculado a partir da salinidade)

$\mathrm{k}_{\mathrm{NH} 4}=$ constante de dissociação do $\mathrm{NH}_{4}^{+}$(medida a partir da variação da temperatura e salinidade) .

\section{ANÁLISES ESTATÍSTICAS}

Os dados log-transformados apresentaram os princípios de parametricidade significativos, incluindo distribuição normal (Kolmogorov-Sminorv, $p<0,05$ ) e homogeneidade de variâncias (Barlett, $\mathrm{p}<0,05$ ). Dessa forma, os dados foram representados por média \pm erro padrão e analisados por testes estatísticos paramétricos (Zar 1989). As variáveis amostradas foram comparadas entre períodos usando ANOVA one-way com medidas repetidas (significativo; 
$\mathrm{p}<0,05)$ seguida do pós-teste de Tukey-Kramer (significativo; $\mathrm{p}<0,05$ ). Além disso, correlações de Pearson (significativo; $\mathrm{p}<0,05$ ) foram usadas para avaliar possíveis relações significativas entre o fluxo de amônia e as demais variáveis estudadas. Todos os testes estatísticos foram realizados usando o programa GraphPrism 4.0.

\section{RESULTADOS}

Todas as estações de coleta apresentaram similares valores, em mediana, de $\mathrm{pH}$, clorofila- $\underline{a}$, nitrogênio dissolvido, nitrogênio total, fósforo dissolvido $\mathrm{e}$ fósforo total, exceto aquela com maior aporte de águas fluviais e efluentes orgânicos (LRF04). Essa estação mais próxima do Jóquei Clube apresentou valores significativamente mais baixos de $\mathrm{pH}$, salinidade, clorofila- $a$ e profundidade, associados a valores sete vezes mais elevados de nutrientes dissolvidos e de $\mathrm{N}$ amoniacal em comparação às demais estações ( $p<0,05$; Tukey-Kramer) (Tabela 1). De acordo com o índice de Salas \& Martino (1991), estação LRF04 também apresentou o único caráter eutrófico, enquanto LRF00,LRF01,LRF05 e LRFJA apresentaram índices similarmente mesotróficos. Já a profundidade total em ambas as estações LRF04 e LRFJA foi mais rasa, refletindo suas posições mais marginais e próximas, respectivamente, dos aportes fluviais e do mar nos extremos opostos da lagoa.

$\mathrm{O} \mathrm{N}$ amoniacal variou entre 0,3 e $44 \mu \mathrm{mol} \mathrm{L}^{-1}$, apresentando valores em mediana (intervalo interquartil 25-75) de $1,1 \quad(0,6-1,8) \mu \mathrm{molL}^{-1}$ ao considerar todas as estações. Na estação LRF04 estes valores foram de $26(18,6-29,3) \mu \mathrm{mol} \mathrm{L}^{-1}$, cerca de 20 vezes mais altos em mediana do que nas demais estações de coleta ( $<<0,05$, Tukey-Kramer; Figura 2). Os subsequentes fluxos de amônia à atmosfera refletiram esse mesmo padrão, valores em mediana cerca de 50 vezes mais elevados em LRF04 do que nas outras estações (Figura 3). Já na comparação entre horários, não foram observadas variações significativas das emissões de amônia ou mesmo das outras variáveis estudadas ( $\mathrm{p}>0,05$; ANOVA one way).

As concentrações de clorofila- $\underline{a}$ confirmaram o estado eutrófico da estação LRF04 (13,2-30mg/L) de acordo com índices para lagos tropicais (Salas \& Martino 1991, Lamparelli 2004). No entanto, não

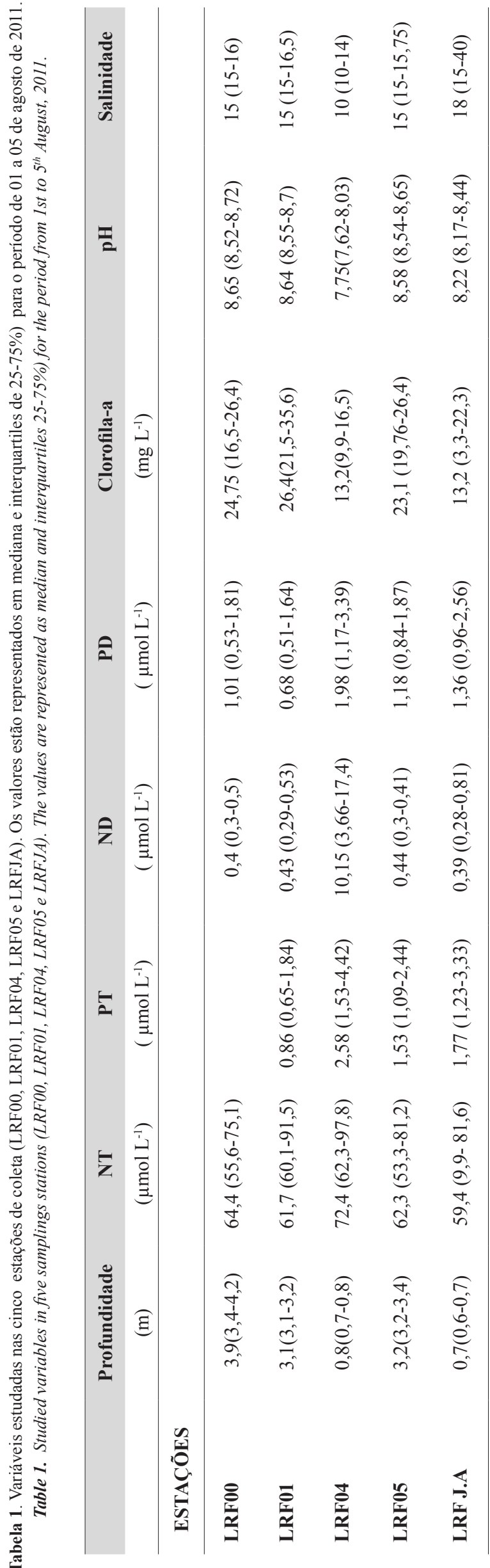


foram observados maiores valores na estação LRF04 em relação às demais parâmetros ( $p>0,05$; TukeyKramer). Já os mais altos valores de salinidade com mediana (intervalo interquartil 25-75) de 18 (15-
40) foram observados nos horários de pico da maré (03:00h e 04:00h) na estação mais próxima ao mar (LRFJA), onde a salinidade era maior nos horários próximos a esse pico de maré e comporta aberta.

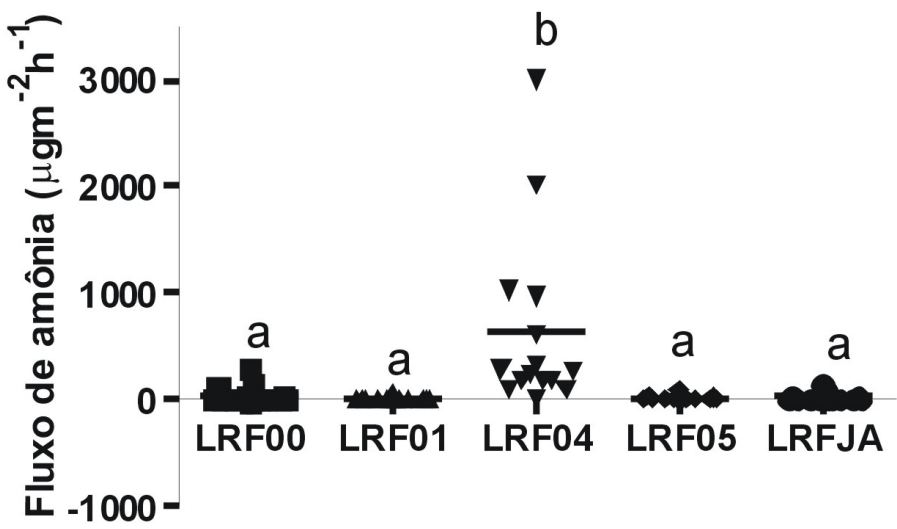

Figura 3. Fluxo de amônia na interface água-ar nas cinco estações (LRF00, LRF01, LRF04, LRF05 e LRFJA) entre o período 01 a 05 de agosto de 2011. O símbolo (-) representa cada amostragem e as letras iguais representam ausência de diferença significativa entre as estações de amostragem ( $\mathrm{p}>0,05$, teste de Tukey-Kramer).

Figure 3. Air-water ammonia fluxes in five sampling stations (LRF00, LRF01, LRF04, LRF05 and LRFJA)from 1st to $5^{\text {th }}$ August, 2011. The symbol (-) represents each sample and the same letters indicate non-significant differences among sampling stations ( $p>0,05$, Tukey-Kramer test).

Na comparação entre os dias consecutivos estudados, os dois últimos (dias 4 e 5) apresentaram mais elevada pluviosidade (dados do INMET/Alerta Rio, Prefeitura do Rio). Estes dias mais chuvosos apresentaram mediana
270 vezes maior em emissão de amônia à atmosfera do que os outros dias (Tukey-Kramer $\mathrm{p}<0,05$ ), passando de $1,2-5,0 \mu \mathrm{g} \mathrm{N} \mathrm{m}{ }^{-2} \mathrm{~h}^{-1}$ nos dias anteriores a $3000-3500 \mu \mathrm{g}$ $\mathrm{N} \mathrm{m}^{-2} \mathrm{~h}^{-1}$ nos dias 4 e 5 (Figura 4).

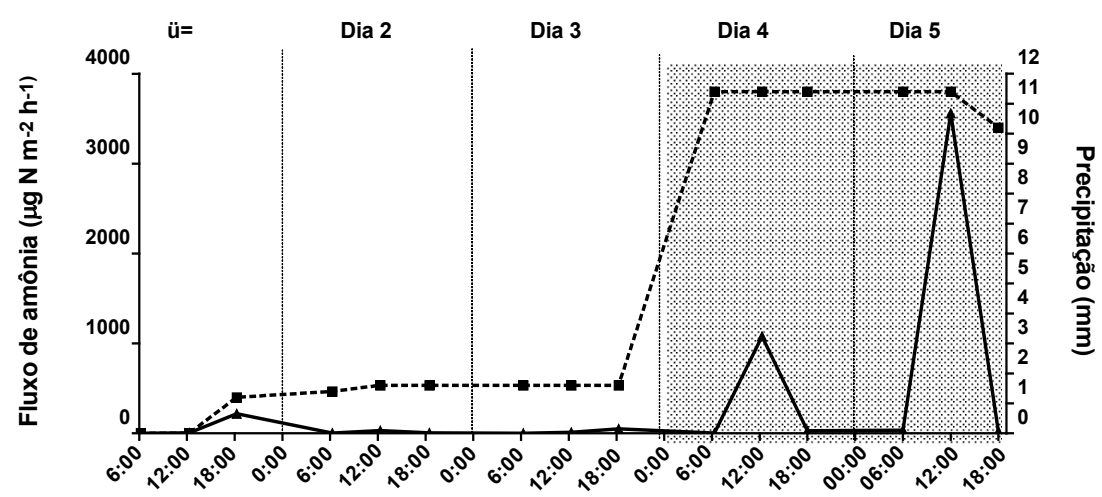

Figura 4. Fluxo de amônia na interface água-ar e precipitação acumulada de 24 h de 1 a 5 de agosto de 2011. Os quadrados representam cada horário. A linha tracejada com quadrados ( $\mathbf{\square})$ indica a precipitação acumulada de 24 horas e a linha sólida com triângulos ( $\boldsymbol{\Delta}$ ), o fluxo de amônia na interface água-ar ( $\mathrm{p}<0,05$, teste de Tukey). A parte hachurada indica os dias chuvosos.

Figure 4. Air-water ammonia fluxes and 24-h accumulated rainfall from 1 st to $5^{\text {th }}$ August, 2011. The triangles represent the sampling time. The dashed line with squares indicates the 24-h accumulated rainfall and the solid lines with triangles the air-water ammonium fluxes over time ( $p<0.05$, Tukey test). The dotted frame indicates rainy days

Em alguns pontos, o fluxo de amônia foi mais elevado (12:00h do dia 4 e 12:00h do dia 5), devido principalmente aos altos valores de emissão da LRF04 em relação ás demais estações (Figura 4).Os resultados evidenciaram uma preponderância na emissão de amônia à atmosfera em todas as estações de coleta, uma vez que $85 \%$ das amostragens no nosso estudo apresentaram supersaturação desse gás. Considerando todas as estações e horários de coleta, os fluxos de $\mathrm{NH}_{3}$ variaram de -10 a $3200 \mu \mathrm{g} \mathrm{N} \mathrm{m}^{-2} \mathrm{~h}^{-1}$ (valores negativos indicam apreensão de $\mathrm{NH}_{3}$ da atmosfera pela superfície da água). Os poucos valores 
negativos de emissão de amônia foram encontrados no dia 5, após às 12:00h, quando observa-se uma redução dos valores de precipitação.

A elevada variabilidade temporal de curto prazo e espacial intralagunar dos fluxos de amônia na interface ar-água (Tabela 2) se refletiu em correlações significativas, especialmente com o $\mathrm{pH}$ e a pluviosidade acumulada de 24 horas (Spearman, $\mathrm{R}>0,60, \mathrm{p}<0,05$; Tabela 2). Os nutrientes dissolvidos (PD e ND), a salinidade e a profundidade apresentaram, por sua vez, baixas correlações significativas com esses fluxos (Spearman, $\mathrm{R}<0.40$, $\mathrm{p}<0.05$; Tabela 2), enquanto os nutrientes totais (PT e NT) e a clorofila- $\underline{a}$ náo apresentaram correlações significativas (Spearman, $\mathrm{p}>0,05$; Tabela 2).

Tabela 2. Correlações de Spearman de cada variável ambiental com os fluxos de amônia na interface água-ar $\left(\mu \mathrm{gN} \mathrm{m}^{-2} \mathrm{~h}^{-1}\right)$ para todas as estações de amostragem e horas durante os cinco dias consecutivos (significativo, $\mathrm{p}<0,05)$. O símbolo ns significa ausência de correlações significativas de Spearman ( $p>0,05)$. Nutrientes estão em $\mu \mathrm{mol} \mathrm{L}{ }^{-1}$, a clorofila- $a$ em mg $\mathrm{L}^{-1}$, a profundidade total em metros $(\mathrm{m})$ e a precipitação acumulada de $24 \mathrm{~h}$ em milímetros ( $\mathrm{mm}$ ). $\mathrm{O}$ pH e salinidade estão em unidades próprias. Table 2. Spearman correlations of each environmental variable with airwater ammonia fluxes $\left(g \mathrm{~N} \mathrm{~m}^{-2} h^{-1}\right)$ for all sampling stations and hours over the 5 consecutive days (significant $p<0.05$ ). The symbol $\underline{n s}$ means non-significant Spearman correlations $(p>0.05)$. Nutrients are in $\mu m o l$ $L^{-1}$, the chlorophyll-a in $\mu g L^{-1}$, the total depth in meters $(m)$ and the 24- $h$ accumulated precipitation in millimeters $(\mathrm{mm})$. The $\mathrm{pH}$ and salinity are in own units.

\begin{tabular}{lc}
\hline Variáveis & Fluxo de Amônia \\
\hline $\mathrm{pH}$ & $-0,63$ \\
Salinidade & $-0,37$ \\
Clorofila-a & $-\mathrm{ns}$ \\
$\mathrm{PT}$ & $\mathrm{ns}$ \\
$\mathrm{NT}$ & $\mathrm{ns}$ \\
PD & 0,31 \\
ND & 0,28 \\
Prof. Total & 0,37 \\
Precipitação & \\
Acumulada & 0,65 \\
\hline
\end{tabular}

\section{DISCUSSÃO}

A consistente predominância de supersaturação e subsequentes emissões de amônia em todos os dias e estações de coleta revelaram o potencial papel dos lagos como fonte de $\mathrm{N}$ para atmosfera, o que sugere também que este $\mathrm{N}$ tem origem na degradação da matéria orgânica e um sítio preferencial à degradação orgânica da bacia de drenagem (Duarte \& Prairie 2005, Cole et al. 2007, Tranvik et al. 2009). Essa predominância da evasão de amônia à atmosfera na lagoa Rodrigo de Feitas também foi associada a uma elevada heterogeneidade na escala espacial intralagunar. Este fenômeno é consequência dos maiores lançamentos pontuais de efluentes orgânicos e inorgânicos na estação LRF04 (Gonzalez et al. 2006), que foi evidenciado com os dados de coliformes totais coletados no mesmo período, o qual demonstrou valores 50 vezes mais elevados de coliformes nesta estação em relação às demais (Van Weerelt et al. 2012, submetido).

A condição mais eutrófica de LRF04 refletiu o papel dos aportes orgânicos diretamente na lagoa ou nos rios contribuintes, especialmente advindo do lançamento de esgotos (Camargo \& Alonso 2006), para aumentar as concentrações tanto de $\mathrm{N}$ amoniacal quanto de outros nutrientes nitrogenados e fosfatados. A intensificação da degradação biológica da matéria orgânica nas águas interiores é reconhecidamente estimulada por esse aporte de recursos orgânicos e inorgânicos (Biddanda \& Cotner 2002) cujos produtos metabólicos incluem gases como o $\mathrm{NH}_{3}$, os quais também podem ser emitidos à atmosfera (Dentener \& Crutzen 1994).

Apesar do curto período de tempo estudado, também foram observadas drásticas mudanças das variáveis limnológicas na comparação entre os dias de coleta. $\mathrm{O}$ aumento da precipitação durante o estudo levou ao incremento nas taxas de emissão de amônia nos dias 4 e 5, resultado da possível entrada de $\mathrm{N}$ nos ecossistemas aquáticos via escoamento e infiltração da bacia de drenagem ou ressuspensão (Guimaraes 2009). Esses resultados confirmaram estudos prévios evidenciando o potencial papel das chuvas em intensificar o carreamento de matéria orgânica e nutrientes do meio terrestre às águas interiores e costeiras (Qasin \& Sengupta 1981, Cotner et al. 2000). Alguns estudos revelam que após um período chuvoso é comum encontrar valores mais elevados de nutrientes dissolvidos, nutrientes totais e produção primária (Ram et al. 2003), evidenciando que existe uma variação entre o período chuvoso e o período seco em ambientes aquáticos.

As chuvas podem aumentar intensamente o aporte de substratos orgânicos à decomposição biológica 
e à subsequente produção de gases ou mesmo de compostos já mineralizados aos ecossistemas lacustres (Rantakari \& Kortelainen 2005), especialmente nas baixas latitudes (Marotta et al. 2010a). Dessa forma, o aporte terrestre intensificado pelas chuvas poderia explicar as mais altas correlações significativas positivas observadas aqui entre os nutrientes e a precipitação acumulada de 24 horas com os dados de todas as estações e dias de coleta da Lagoa Rodrigo de Freitas. A própria água da chuva pode carrear amônia (Migon et al. 1991, Lima et al. 2009), especialmente em áreas urbanas (Ugucione et al. 2002)(Ugucione et al. 2002), constituindo uma fonte adicional para o aumento destes compostos nos ecossistemas aquáticos. As estimativas de emissão de amônia à atmosfera na Lagoa Rodrigo de Freitas nos dias chuvosos foram altas, uma vez que superaram cerca de cinco vezes aqueles encontrados na Baia da Guanabara, que se localiza a poucos km da Lagoa, com elevada carga de esgotos (Guimaraes $\&$ de Mello 2006). Nesse sentido, os dias secos apresentaram inclusive valores de fluxo negativo, indicando entrada de $\mathrm{N}$ atmosférico para a superfície da água e confirmando este potencial da chuva sobre o aporte alóctone de $\mathrm{N}$ amoniacal.

As altas temperaturas tropicais em associação a diferenças espaciais e temporais de origem natural ou antropogênica podem ter contribuído para a elevada variabilidade das emissões de amônia na lagoa costeira estudada. Como conclusão, nossos resultados indicaram que dinâmicas antropogênicas, como o lançamento de efluentes e as aberturas da ligação ao mar, podem contribuir para a heterogeneidade intralagunar e substanciais mudanças de curto prazo nas variáveis limnológicas em uma lagoa tropical. Esse elevado dinamismo frente alterações humanas, especialmente intensificado por condições naturais tropicais advindas do variável aporte dos ambientes marinho, dulcícola e terrestre, demanda ser melhor considerado em eventuais ações de monitoramento e manejo nesses ecossistemas. O papel dos aportes antropogênicos de matéria orgânica e nutrientes nos ecossistemas aquáticos pode representar uma importante contribuição à emissão de gases com a propriedade de intensificar a acidificação e o aquecimento global na superfície terrestre, uma lacuna científica crucial a ser ainda mais bem compreendida no âmbito da bacia de drenagem.
AGRADECIMENTOS: Os dados desse capítulo foram gerados durante a disciplina Biogeoquímica Experimental, oferecida pelo Programa de Pós Graduação em Ecologia da UFRJ. Agradecemos ao CNPq, CAPES e FAPERJ pelo financiamento dessa pesquisa, que é parte da dissertação de mestrado da primeira autora. Os autores são gratos ao pescador Orlando Marins pelo apoio logístico e ajuda nas coletas. Agradecemos também ao Clube Piraquê que nos acolheu em seu espaço durante uma semana de coleta, ao Instituto Nacional de Meteorologia pelos dados de pluviosidade e ao Instituto Estadual de Meio Ambiente pelos registros de abertura de comportas fluviais e do Jardim de Alah.

\section{REFERÊNCIAS}

APHA. 1992. Standard methods for the examination of water and wastewater. 18ed. Byrd Prepess Spingfield, Washington, DC. 1155p.

ARAUJO, C.L. 2008. Análise da concentração de mercúrio no fitobentos da Lagoa Rodrigo de Freitas, RJ. Monografia. Universidade Estadual do Rio de Janeiro, Rio de Janeiro, RJ. 54 p. ASMAN, W.A.H.; SUTTON, M.A. \& SCHJORRING, J.K. 1998. Ammonia: emission, atmospheric transport and deposition. New Phytologist, 139: 27-48, http:/dx.doi.org/ 10.1046/j.14698137.1998.00180.x

BIDDANDA, B.; OGDAHL, M. \& COTNER, J. 2001. Dominance of bacterial metabolism in oligotrophic relative to eutrophic waters. Limnology and Oceanography, 46 (3): 730739, http://dx.doi.org/ 0.4319/1o.2001.46.3.0730

BOUWMAN, A.F.; LEE, D.S.; ASMAN, W.A.H.; DENTENER, F.J.; VANDERHOEK, K.W. \& OLIVIER, J.G.J. 1997. A global high-resolution emission inventory for ammonia. Global Biogeochemical Cycles, 11: 561-587, http://dx.doi. org/10.1029/2011GB004161

CAMARGO, J.A. \& ALONSO, A. 2006. Ecological and toxicological effects of inorganic nitrogen pollution in aquatic ecosystems: A global assessment. Environment International, 32: 831-849, http://dx.doi.org/ 10.1016/j.envint.2006.05.002

COLE, J.J.; PRAIRIE, Y.T. \& CARACO, N.F. 2007. Plumbing the global carbon cycle: Integrating inland waters into the terrestrial carbon budget. Ecosystems, 10: 171-184, http://dx.doi. org/10.1007/s10021-006-9013-8

COTNER, J.B.; JOHENGEN, T.J. \& BIDDANDA, B.A. 2000. Intense winter heterotrophic production stimulated by benthic resuspension. Limnology and Oceanography, 45: 1672-1676, http://dx.doi.org/10.4319/1o.2000.45.7.1672

DENTENER, F.J. \& CRUTZEN, P. J. 1994. A 3-Dimensional Model of the Global Ammonia Cycle. Journal of Atmospheric Chemistry, 19: 331-369, http://dx.doi.org/ 10.1007/BF00694492 
DUARTE, C.M. \& PRAIRIE, Y.T. 2005. Prevalence of heterotrophy and atmospheric $\mathrm{CO} 2$ emissions from aquatic ecosystems. Ecosystems, 8: 862-870, http://dx.doi.org/10.1007/ s10021-005-0177-4

ESTEVES, F.A. 1998. Lagoas Costeiras: Origem, funcionamento e possibilidades de manejo. Pp. 63-87. In: Ecologia de Lagoas Costeiras. Macaé, RJ. 464p.

FEEMA (Fundação Estadual de Engenharia do Meio Ambiente). 2006. Lagoa Rodrigo de Freitas: diagnóstico de qualidade da água. Secretária do Estado de Meio Ambiente e Desenvolvimento Urbano, Rio de Janeiro, RJ.

FELIX, E.P. \& CARDOSO, A.A. 2004. Atmospheric ammonia: Sources, transformation, sinks, and methods of analysis. Química Nova, 27: 123-130, http://dx.doi.org/10.1590/S0100

FENCHEL, T. 2008. The microbial loop-25 years later. Journal of Experimental Marine Biology and Ecology, 366: 99-103, http://dx.doi.org/ 10.1016/j.jembe.2008.07.013

GONZALEZ, A.M.; PARANHOS, R. \& LUTTERBACH, M.S. 2006. Heterotrophic bacteria abundances in Rodrigo de Freitas Lagoon (Rio de Janeiro, Brazil). Brazilian Journal of Microbiology, 37: 428-433,http://dx.doi.org/ 10.1590/S151783822006000400005

GUIMARAES, G.P. \& DE MELLO, W.Z. 2006. Estimate of ammonia fluxes at the air-sea interface in the Guanabara Bay A preliminary study. Química Nova, 29: 54-60,http://dx.doi.org/ 10.1590/S0100-40422006000100012

JOHNSON, M.T.; LISS, P.S.; BELL, T.G.; LESWORTH, T.J.; BAKER, A.R.; HIND, A.J.; JICKELLS, T.D.; BISWAS, K.F.; WOODWARD, E.M.S. \& GIBB, S.W. 2008. Field observations of the ocean-atmosphere exchange of ammonia: Fundamental importance of temperature as revealed by a comparison of high and low latitudes. Global Biogeochemical Cycles, 22: GB1019, http://dx.doi.org/ 0.1029/2007GB003039

KJERFVE，B.R. \& MAGILL, K.E. 1989. Geographic and hydrodynamic characteristics of shallow coastal lagoons. Marine Geology, 88: 187-199, http://dx.doi.org/10.1016/00253227(89)90097-2

KOLASA, J. \& ROLLO, C.D. 1991. Introduction: the heterogeneity of heterogeneity: a glossary. Pp. 1-23. In: J. Kolasa \& S.T.A. Pickett (eds.). Ecological Heterogeneity. SpringerVerlag, New York, NY, USA. 332p.

LAMPARELLI, M.C. 2004. Grau de trofia em corpos d'água do estado de São Paulo: avaliação dos métodos de monitoramento. Tese de Doutorado. Universidade de São Paulo, São Paulo, SP. 238p.
LEAL, J.J.F.; ENRICH-PRAST, A.; ESTEVES, F.D.; BOZELLI, R. \& FARJALLA, V.F. 2005. Influence of Campsurus notatus bioturbation on oxygen profile and uptake in sediments of an Amazonian lake impacted by bauxite tailings. Archiv Fur Hydrobiologie, 162: 557-574,http://dx.doi.org/10.1127/00039136/2005/0162-0557

LIMA, R.G; CAVALCANTE, P.R.S.; MELO,O.T;MELLO, W.Z. 2009. Concentração de amônio na água da chuva e estimativas de emissão de amônia de rebanhos domésticos de Pinheiro e Viana, Baixada Maranhense. Química Nova, 32: 2273-2276, http:// dx.doi.org/10.1590/S0100-40422009000900006

LOUREIRO, D.D. 2006. Distribuição espacial do mercúrio nos sedimentos superficiais da Lagoa Rodrigo de Freitas. In: III Simpósio Brasileiro de Oceanografia, São Paulo, SP, Brasil.

MACHADO, L.B. 2008. Evolução da Degradação Ambiental da Bacia Hidrográfica da Lagoa Rodrigo de Freitas. Dissertação de Mestrado. Universidade do Estado do Rio de Janeiro. Rio de Janeiro, RJ, Brasil. 178p.

MAROTTA, H.; DUARTE, C.M.; MEIRELLES-PEREIRA, F.; BENTO, L.; ESTEVES, F.A. \& ENRICH- MAROTTA, H.; SANTOS, R. O. \& ENRICH-PRAST, A. 2008. Monitoramento limnológico: um instrumento para a conservação dos recursos hídricos no planejamento e na gestão urbano-ambientais. Ambiente e Sociedade, 11: 67-79, http://dx.doi.org/10.1590/ S1414-753X2008000100006

MAROTTA, H.; DUARTE, C.M.; SOBEK, S. \& ENRICHPRAST, A. 2009. Large CO2 disequilibria in tropical lakes. Global Biogeochemical Cycles, 23: GB4022,http://dx.doi.org/ 10.1029/2008GB003434

MAROTTA, H.; DUARTE, C.M.; PINHO, L. \& ENRICHPRAST, A. 2010. Rainfall leads to increased $\mathrm{pCO}_{2}$ in Brazilian Coastal Lakes. Biogeosciences, 6:11521-11539, http://dx.doi. org/10.5194/bg-7-1607-2010

MAROTTA, H.; DUARTE, C.M.; MEIRELLES-PEREIRA, F.; BENTO, L.; ESTEVES, F.A. \& ENRICH-PRAST, A. 2010 b. Long-Term CO2 Variability in Two Shallow Tropical Lakes Experiencing Episodic Eutrophication and Acidification Events. Ecosystems, 13: 382-392, http://dx.doi.org/ 0.1007/s10021-0109325-6.

MIGON, C.; MORELLI, J.; NICOLAS, E. \& COPIN-MONT_ EGUT, G. 1991. Evaluation of total atmospheric deposition of $\mathrm{Pb}, \mathrm{Cd}$, Cuand $\mathrm{Zn}$ to the Ligurian Sea. The Science of the Total Environment 105, 135-148,http://dx.doi.org/10.1016/00489697(91)90336-D 
MORRIS, J. T. \& P. M. BRADLEY. 1999. Effects of nutrient loading on the carbon balance of coastal wetland sediments. Limnology and Oceanography, 44: 699-702, http://dx.doi.org/ 10.4319/1o.1999.44.3.0699.

NUSCH, E. A. \& G. PALME. 1975. Biologische Methoden für die Praxis der Gewässeruntersuchung. Bestimmung des Chlorophyll-a und Phaeopigmentgehaltes in Oberflächenwasser. GWF-Wasser/Abwasser 116: 562-565.

OLIVEIRA, J.A. 1976. Contribuição ao conhecimento da fauna da Lagoa Rodrigo de Freitas. SudepeI, Rio de Janeiro, RJ. 11p.

PEREIRA, R.M.V. \& MEDEIROS, R. 2009. A aplicação dos instrumentos do sistema de gestão e do sistema de gerenciamento dos recursos hídricos na Lagoa Rodrigo de Freitas, RJ, Brasil. Ambi-água, 4: 211-229,http://dx.doi.org/10.4136/ambi-agua.113.

QASIM, S. Z., AND R. SENGUPTA. 1981. Environmental characteristics of the Mandovi-Zuari Estuarine System in Goa. Estuaries Coastal Shelf Science., 13: 557-578, http://dx.doi.org/ 10.1016/S0302-3524(81)80058-8

QUINN, P.K.;BARRETT, K.J.;DENTENER, F.J.;LIPSCHULTZ, F. \& SIX, K.D. 1996. Estimation of the air/sea exchange of ammonia for the North Atlantic basin. Biogeochemistry 35,275304,http://dx.doi.org/ 10.1007/BF02179831

RAM, A.S.P.; NAIR, S. \& CHANDRAMOHAN, D. 2003. Seasonal shift in net ecosystem production in a tropical estuary. Limnology and Oceanography, 48: 1601-1607, http://dx.doi.org/ 10.4319/lo.2003.48.4.1601

RANTAKARI，M. \& KORTELAINEN，P. 2005. Interannual variation and climatic regulation of the $\mathrm{CO} 2$ emission from large boreal lakes. Global Change Biology, 11: 1368-1380,http:// dx.doi.org/ 10.1111/j.1365-2486.2005.00982.x

SALAS, H.J. \& MARTINO, P. 1991. A SIMPLIFIED PHOSPHORUS TROPHIC STATE MODEL FOR WARMWATER TROPICAL LAKES. Water Research, 25: 341-350, http://dx.doi.org/ 10.1016/0043-1354(91)90015-I

SCHINDLER, D.E.; CARPENTER, S.R.; COLE, J.J.; KITCHELL, J.F. \& PACE, M.L. 1997. Influence of food web structure on carbon exchange between lakes and the atmosphere. Science, 277: 248251,http://dx.doi.org/10.1126/science.277.5323.248

SCHINDLER, D.W. 2006. Recent advances in the understanding and management of eutrophication. Limnologyand Oceanography, 51: 356-363, http://dx.doi.org/ 0.4319/1o.2006.51.1_part_2.0356
STAEHR, P. A. \& SAND-JENSEN, K. 2007. Temporal dynamics and regulation of lake metabolism. Limnology and Oceanography, 52: 108-120, http://dx.doi.org/10.4319/1o.2007.52.1.0108

SUTTON, M.A.; MILFORD, C.; DRAGOSITS, U.; PLACE, C.J.; SINGLES, R.J.; SMITH, R.I.; PITCAIRN, C.E.R.; FOWLER, D.; HILL, J.; APSIMON, H.M.; ROSS, C.; HILL, R.; JARVIS, S.C.; PAIN, B.F.; PHILLIPS, V.C.; HARRISON, R.; MOSS, D.; WEBB, J.; ESPENHAHN, S.E.; LEE, D.S.; HORNUNG, M.; ULLYETT, J.; BULL, K.R.; EMMETT, B.A.; LOWE, J. \& WYERS, G.P. 1998. Dispersion, deposition and impacts of atmospheric ammonia: quantifying local budgets and spatial variability. Environmental Pollution, 102: 349-361, http:// dx.doi.org/ 10.1016/S0269-7491(98)80054-7

TRANVIK, L.J.; DOWNING, J.A.; COTNER, J.B.; LOISELLE, S.A.; STRIEGL， R.G.; BALLATORE，T.J.; DILLON, P.; FINLAY, K.; FORTINO, K.; KNOLL, L.B.; KORTELAINEN, P.L.; KUTSER, T.; LARSEN, S.; LAURION, I.; LEECH, D.M.; MCCALLISTER, S.L.; MCKNIGHT, D.M.; MELACK, J.M.; OVERHOLT, E.; PORTER, J.A.; PRAIRIE, Y.; RENWICK, W.H.; ROLAND, F.; SHERMAN, B.S.; SCHINDLER, D.W.; SOBEK, S.; TREMBLAY, A.; VANNI, M.J.; VERSCHOOR, A.M.; VON WACHENFELDT, E. \& WEYHENMEYER, G.A. 2009. Lakes and reservoirs as regulators of carbon cycling and climate. Limnology and Oceanography, 54: 2298-2314, http:// dx.doi.org/ 10.4319/lo.2009.54.6 part 2.2298

TUNDISI, J. G. 1997. A note on the effect of rainfall in the process of stratification and stability in the Rio Doce lakes. Pp. 79-82. In: J.G. Tundisi \& Y. Saijo (eds.). Limnological Studies on the Rio Doce Valley Lakes, Brazil. Brazilian Academy of Sciences.

UGuCiOne, C., E. P. FELIX, G. O. DA ROCHA, AND A. A. CARDOSO. 2002. Daytime and nighttime removal processes of atmospheric $\mathrm{NO} 2$ and $\mathrm{NH} 3$ in Araraquara's region SP. Ecletica Quimica 27: 103-112,http://dx.doi.org/10.1590/S010046702002000200009.

VITOUSEK, P. M.; ABER, J.D.; HOWARTH, R.W.; LIKENS, G.E.; MATSON, P.A.; SCHINDLER, D.W.; SCHLESINGER, W.H. \& TILMAN, G.D. 1997. Human alteration of the global nitrogen cycle: Sources and consequences. Ecological Applications, 7: 737-750, http://dx.doi.org/ 10.2307/2269431

YANNARELL, A.C.; KENT, A.D.; LAUSTER, G.H.; KRATZ, T.K. \& TRIPLETT, E.W. 2003. Temporal patterns in bacterial communities in three temperate lakes of different trophic status. Microbial Ecology, 46:391-405,http://dx.doi.org/10.1007/s00248003-1008-9 
ZAR, J.H. 1989. Statistical procedures for biological-research A citation classic commentary on biostatistical analysis by Zar, J.H. Current Contents/Agriculture Biology \& Environmental Sciences, 6: 20-20.

Submetido em 09/02/2012

Aceito em 11/07/2012 